\title{
Utilidad de la amilasa y la lipasa séricas como predictores de gravedad en la pancreatitis poscolangiopancreatografía retrógrada endoscópica
}

\author{
Usefulness of serum amylase and lipase as predictors of the severity of post-endoscopic \\ retrograde cholangiopancreatography pancreatitis
}

\author{
Uriel Martínez-Segundo ${ }^{*}$, Víctor M. Pinto-Angulo", Javier García-Álvarez², Juan M. Cruz-Reyes ${ }^{1}$ y \\ Giuseppe Briceño-Sáenz' \\ 'Servicio de Cirugía General; ²Sociedad Médico Quirúrgica Hospital Juárez de México, Ciudad de México, México
}

\begin{abstract}
Resumen
Antecedentes: La pancreatitis poscolangiopancreatografía retrógrada endoscópica (PPCPRE) es la complicación más frecuente de este procedimiento. Objetivo: Demostrar si la amilasa y la lipasa séricas se correlacionan con la presencia y la gravedad de la PPCPRE. Método: Realizamos un estudio retrospectivo, observacional y analítico de pacientes a quienes se realizó CPRE. Los que desarrollaron pancreatitis se clasificaron por gravedad de acuerdo con la revisión de Atlanta de 2012 y se analizaron sus concentraciones séricas de enzimas. Empleamos curvas ROC (Receiver Operating Characteristics) para conocer los mejores puntos de corte enzimáticos y analizamos sus rendimientos diagnósticos. Usamos las pruebas de ji al cuadrado, $t$ de Student y $U$ de Mann Whitney para el análisis de las variables, y se consideró estadísticamente significativo un valor de $p<0.05$. Resultados: De un total de 621 pacientes, 54 presentaron pancreatitis. Para pancreatitis moderadamente grave y grave, unas cifras de lipasa de $1500 \mathrm{U} / \mathrm{I}$ presentaron un área bajo la curva $(A \cup C)=0.827$ (intervalo de confianza del 95\% [IC 95\%]: 0.67-0.98), con una sensibilidad del 72.7\%, una especificidad del $86 \%$ y un valor predictivo negativo del 92.5\% ( $p<0.05$ ); y unas cifras de amilasa de $920 \mathrm{U} / \mathrm{I}$ presentaron un $A U C=0.65$ (IC 95\%: 0.43-0.86), con una sensibilidad del $63 \%$ y una especificidad del $67 \%$ ( $p>0.05$ ). Conclusiones: La lipasa muestra correlación con la presencia y la gravedad de la PPCPRE. La amilasa muestra correlación no significativa con la PPCPRE.
\end{abstract}

Palabras clave: Amilasa. Colangiopancreatografía retrógrada endoscópica. CPRE. Lipasa. Pancreatitis. PPCPRE.

\begin{abstract}
Background: Post-ERCP pancreatitis (PEP) is the most common complication of Post-endoscopic retrograde cholangiopancreatography. Objective: to demonstrate whether serum amylase and lipase values correlate with the presence and severity of PEP. Method: We conducted a retrospective, observational and analytical study of patients who underwent ERCP, those who developed pancreatitis were classified by severity according to the 2012 revised Atlanta criteria and their serum enzyme levels were analyzed. We used ROC (Receiver Operating Characteristics) curves to know the best enzyme cutoff points and
\end{abstract}

\section{Correspondencia:}

*Uriel Martínez-Segundo

Avda. Politécnico Nacional, 5160

Col. Magdalena de las Salinas, Del. Gustavo A. Madero

C.P. 07760 Ciudad de México, México

E-mail: ums_18@ @otmail.com

Fecha de recepción: 07-11-2019

Fecha de aceptación: 08-01-2020

DOI: $10.24875 / C I R U .20001691$
Cir Cir. 2020;88(4):428-434

Contents available at PubMed www.cirugiaycirujanos.com 0009-7411/@ 2020 Academia Mexicana de Cirugía. Publicado por Permanyer. Este es un artículo open access bajo la licencia CC BY-NC-ND (http://creativecommons.org/licenses/by-nc-nd/4.0/). 
analyzed their diagnostic yields. Chi-square, $t$-distribution and Mann-Whitney $U$ test were used in the variable analysis and it was considered statistically significant when $p<0.05$. Results: A total 621 patients, 54 presented pancreatitis. For moderately severe and severe forms: lipase level of $1500 \mathrm{U} / \mathrm{L}$ had an area under the curve $(A U C)=0.827,95 \% \mathrm{Cl}(0.67-0.98)$, sensitivity $=72.7 \%$, specificity $=86 \%$, negative predictive value $=92.5 \%, p<0.05$. Amylase level of $920 \mathrm{U} / \mathrm{L}$ presented AUC $=0.65,95 \% \mathrm{Cl}(0.43-0.86)$, sensitivity $=63 \%$, specificity $=67 \%, p>0.05$. Conclusions: Serum lipase shows correlation with the presence and severity of PEP. Amylase shows no significant correlation with PEP.

Key words: Amylase. Endoscopic retrograde cholangiopancreatography. ERCP. Lipase. Pancreatitis. PEP.

\section{Introducción}

La pancreatitis aguda es la complicación más frecuente después de una colangiopancreatografía retrograda endoscópica (CPRE). Su frecuencia de presentación es muy variable, pero habitualmente se reporta entre el 3 y el $10 \%$.

El mecanismo por el que se presenta la pancreatitis pos-CPRE (PPCPRE) no está del todo comprendido, pero se sugiere que podría ser multifactorial ${ }^{2,3}$. Se piensa que podría representar una pancreatitis aguda diferente de la ocasionada por otras etiologías, con base en el patrón de la elevación de las enzimas pancreáticas, las manifestaciones clínicas y el peor pronóstico cuando se presenta en su forma grave ${ }^{3,4}$.

Los grandes estudios han identificado factores de riesgo que se relacionan con la presencia de PPCPRE, que pueden clasificarse como factores relacionados con el paciente, con el procedimiento y con el operador ${ }^{1}$.

Se describe que la mayoría de los episodios de PPCPRE son leves (alrededor de 90\%) y el resto desarrollan pancreatitis moderada o grave (alrededor de 10\%).

Existen varios métodos que intentan reducir la frecuencia de PPCPRE, como emplear técnicas de canulación que minimicen el trauma ampular, usar stents del conducto pancreático, administración rectal de antiinflamatorios no esteroideos (AINE) o protocolos específicos de hidratación intravenosa'. Sin embargo, aun con el empleo de dichas medidas es difícil prevenir completamente esta complicación en particular ${ }^{6}$.

Existen escalas de predicción del desarrollo de PPCPRE basadas en los factores de riesgo relacionados con su presentación ${ }^{7-9}$, pero contemplan demasiadas variables y esto las hace poco prácticas.

Solo hay algunos estudios específicamente en pacientes sometidos a CPRE que evalúan la utilidad de la determinación de la amilasa y la lipasa séricas para predecir la gravedad de la pancreatitis ${ }^{2,6,10-12}$, los cuales muestran que las elevaciones de sus cifras se correlacionan con la presencia de formas graves de pancreatitis y pueden emplearse como predictores; sin embargo, las cifras de corte, los criterios de clasificación de gravedad y el rendimiento diagnóstico difieren.

La cuantificación de la amilasa y la lipasa puede encontrarse disponible en los hospitales de nuestro país, y llevar a cabo un estudio para evaluar la utilidad de dichas enzimas en la PPCPRE podría ser de gran importancia, ya que, si se demuestra alguna utilidad, contaríamos con una herramienta diagnóstica que podría ser aplicable a nuestra población y que nos daría pauta para la toma de mejores decisiones clínicas.

El objetivo de este estudio es demostrar si los valores de amilasa y de lipasa se correlacionan con la presencia y la gravedad de la PPCPRE.

\section{Método}

Realizamos un estudio retrospectivo, observacional y analítico, en el Hospital Juárez de México, de pacientes hospitalizados a quienes se realizó CPRE en el periodo comprendido de marzo de 2016 a febrero de 2019. Los criterios de inclusión fueron los siguientes: pacientes con CPRE programada o urgente en el mismo hospital, aplicación de indometacina, permanencia en hospitalización después de la CPRE, contar con expediente completo y disponer de registros de amilasa o lipasa después del procedimiento. Los criterios de no inclusión fueron: pacientes menores de 18 años, referidos de otro hospital, antecedente de pancreatitis, pacientes oncológicos y aquellos que presentaron otras complicaciones diferentes de la pancreatitis o que se derivan de esta.

La administración de indometacina fue por vía rectal, en dosis única de $100 \mathrm{mg}$, estando el paciente ya en la sala y al inicio del procedimiento. La toma de la muestra sanguínea para cuantificar la amilasa y la lipasa se lleva a cabo, de forma sistemática, el día en que se realiza el procedimiento a las 18:00 horas; considerando el horario en que habitualmente se hacen 
los procedimientos, implica que entre la realización de la CPRE y la toma de la muestra sanguínea para su análisis trascurre un tiempo de 4 a 8 horas.

Empleamos la clasificación y las definiciones de Atlanta $^{13}$ para el diagnóstico y la categorización de la gravedad de la pancreatitis.

Se dividió en dos grupos a la población estudiada, de acuerdo con la presentación de la pancreatitis. Determinamos la frecuencia de PPCPRE. Se clasificó a los pacientes según la pancreatitis en leve, moderadamente grave y grave, y a continuación en dos grupos: uno con los que presentaron la forma leve y otro con los que presentaron las formas graves, es decir, aquellos con pancreatitis moderadamente grave o grave. Comparamos los valores de amilasa y de lipasa entre ambos grupos. Los datos obtenidos los resumimos como porcentajes, medianas, percentiles, medias y desviación estándar. Para el análisis de las variables cualitativas empleamos la prueba de ji al cuadrado, y para las variables continuas empleamos las pruebas t de Student y U de Mann Whitney, dependiendo de su distribución normal o libre. Las pruebas estadísticas empleadas fueron a dos colas y se consideró estadísticamente significativo un valor de $p<0.05$.

Empleamos curvas ROC (Receiver Operating Characteristics) para conocer los puntos de corte de los valores de amilasa y de lipasa como predictores de las formas graves de pancreatitis. Determinamos la sensibilidad, la especificidad, el valor predictivo positivo y el valor predictivo negativo de los valores de corte encontrados para cada enzima en la predicción de las formas graves. Para el análisis de los datos empleamos el programa SPSS versión 25 para Windows.

\section{Resultados}

Un total de 621 pacientes reunieron los criterios de inclusión del presente estudio, con una proporción de mujeres y hombres de 2.1:1. De todos los pacientes, 567 no presentaron pancreatitis y 54 sí la presentaron, por lo que la frecuencia de esta complicación es del $8.7 \%$. El diagnóstico de PPCPRE se hizo por criterio clínico más criterio de laboratorio en 52 pacientes, y por criterio clínico más complicación local por imagen (tomografía) de manera retrospectiva en dos pacientes.

En la tabla 1 se comparan las principales características de los pacientes con y sin pancreatitis.

Al estudiar la variable dolor abdominal encontramos que todos los pacientes con pancreatitis desarrollaron dolor abdominal, definido como
Tabla 1. Características de los pacientes con y sin pancreatitis

\begin{tabular}{|c|c|c|c|}
\hline & \multicolumn{2}{|c|}{ PPCPRE } & \multirow[t]{2}{*}{$p$} \\
\hline & Sí & No & \\
\hline $\begin{array}{l}\text { Número de } \\
\text { pacientes (\%) }\end{array}$ & $54(8.7)$ & $567(91.3)$ & \\
\hline Edad & 43.5 años $^{*}\left(28^{\dagger}\right.$ a $\left.59^{\ddagger}\right)$ & 45 años $^{*}\left(31^{\dagger}\right.$ a $\left.61^{\ddagger}\right)$ & 0.6 \\
\hline Sexo $(\%)$ & $\begin{array}{l}37 \text { mujeres }(68.5) \\
17 \text { hombres }(31,5)\end{array}$ & $\begin{array}{c}383 \text { mujeres (67.5) } \\
184 \text { hombres (32.5) }\end{array}$ & \\
\hline $\begin{array}{l}\text { Índice de masa } \\
\text { corporal }\end{array}$ & $28^{\S}\left(3.1^{11}\right)$ & $27.37^{\S}\left(2.94^{\Uparrow}\right)$ & 0.2 \\
\hline $\begin{array}{l}\text { Principales } \\
\text { indicaciones } \\
\text { de solicitud del } \\
\text { procedimiento, } \\
\text { número de } \\
\text { pacientes (\%) }\end{array}$ & $\begin{array}{c}\text { PB } \\
\text { Coledocolitiasis, } 35 \\
(64.8) \\
\text { Colangitis, } 11(20.3) \\
\text { Fístula biliar, } 5 \text { (9.3) }\end{array}$ & $\begin{array}{c}\text { PB } \\
\text { Coledocolitiasis, } 383 \\
(67.5) \\
\text { Colangitis, } 92(16) \\
\text { Fístula biliar, } 31(5.5)\end{array}$ & \\
\hline \multicolumn{4}{|c|}{$\begin{array}{l}\text { *Mediana. } \\
\text { †Percentil } 25 . \\
\text { †Percentil } 75 . \\
\text { sMedia. } \\
\text { 'Desviación estándar. } \\
\text { Nota: ambos grupos de pacientes son muy homogéneos en cuanto a edad y sexo, } \\
\text { así como en los principales motivos de solicitud de CPRE. Hay una ligera tendencia } \\
\text { a un mayor índice de masa corporal en los que presentaron pancreatitis, pero sin } \\
\text { significancia estadística. } \\
\text { PB: Probable coledocolitiasis; PPCPRE: pancreatitis poscolangiopancreatografía } \\
\text { retrógrada endoscópica. }\end{array}$} \\
\hline
\end{tabular}

dolor epigástrico que se irradia a espalda, de nuevo inicio o exacerbación de dolor previo. Cabe destacar que el $21.3 \%$ de los pacientes que no desarrollaron pancreatitis también presentaron dolor abdominal después del procedimiento; con la prueba de ji al cuadrado confirmamos que dichas diferencias observadas son estadísticamente significativas $(p<0.05)$.

El $100 \%$ de las muestras sanguíneas para cuantificación de las enzimas pancreáticas se tomaron entre 4 y 8 horas tras la realización de la CPRE. Del total de los pacientes, el $92.4 \%$ contaron con mediciones tanto de amilasa como de lipasa. El $97 \%$ de los reportes de los resultados de dichas enzimas se obtiene en un lapso menor de 12 horas a partir de realizada la CPRE, y el $100 \%$ antes de las 17 horas.

Las características de las enzimas pancreáticas en los grupos con y sin pancreatitis se muestran en la tabla 2.

En el grupo de pacientes sin pancreatitis, la elevación enzimática de al menos tres veces el límite superior normal se presentó en el $33.5 \%$ y el $14.5 \%$ para la amilasa y la lipasa, respectivamente.

Al clasificar a los pacientes con pancreatitis según la gravedad obtuvimos que $43(79.6 \%)$ corresponden a pancreatitis leves, $9(16.7 \%)$ a moderadamente graves y $2(3.7 \%)$ a graves. 
Tabla 2. Características de las enzimas pancreáticas en la población de estudio

\begin{tabular}{|c|c|c|c|c|}
\hline & \multicolumn{2}{|c|}{$\begin{array}{l}\text { Pacientes con } \\
\text { PPCPRE }(n=54)\end{array}$} & \multicolumn{2}{|c|}{$\begin{array}{c}\text { Pacientes sin } \\
\text { PPCPRE }(n=567)\end{array}$} \\
\hline & $\begin{array}{c}\text { Amilasa } \\
(\mathrm{U} / \mathrm{I})\end{array}$ & $\begin{array}{l}\text { Lipasa } \\
\text { (U/I) }\end{array}$ & $\begin{array}{c}\text { Amilasa } \\
(\mathrm{U} / \mathrm{I})\end{array}$ & $\begin{array}{c}\text { Lipasa } \\
\text { (U/I) }\end{array}$ \\
\hline Media & 925 & 1253 & 156 & 96 \\
\hline Mediana & 860 & 1180 & 105 & 58 \\
\hline Desviación estándar & 519 & 658 & 171 & 129 \\
\hline Mínimo & 152 & 199 & 7 & 7 \\
\hline Máximo & 2997 & 3190 & 2050 & 1270 \\
\hline Percentil 25 & 593 & 835 & 60 & 35 \\
\hline Percentil 75 & 1063 & 1503 & 188 & 99 \\
\hline
\end{tabular}

en el grupo con PPCPRE, 52 pacientes ambas enzimas y 2 solo lipasa; en el grupo sin PPCPRE, 5 solo amilasa, 40 solo lipasa y 522 ambas enzimas. Hubo una tendencia a mayores niveles enzimáticos en el grupo con PPCPRE. Las diferencias observadas en las mediciones de cada enzima entre los dos grupos son estadísticamente significativas, con $\mathrm{p}<0.05$

PPCPRE: pancreatitis poscolangiopancreatografía retrógrada endoscópica.

Al dividir en dos grupos a los pacientes con pancreatitis, las enzimas presentan mayor elevación en las formas moderadamente grave y grave que en la forma leve (Fig. 1). Al analizar estas diferencias entre ambos grupos se observa que poseen una distribución libre y, por lo tanto, al emplear la prueba $U$ de Mann Whitney se confirma que la diferencia observada para el caso de la amilasa no es estadísticamente significativa $(p=0.052)$. No obstante, en el caso de la lipasa se comprueba que si existe una correlación: a mayor elevación enzimática, mayor gravedad de la PPCPRE, resultado que es estadísticamente significativo $(p<0.05)$.

Las curvas ROC de cada enzima para la predicción de pancreatitis grave y moderadamente grave se muestran en las figuras 2 y 3 .

El área bajo la curva (AUC) de la curva ROC de la amilasa es de 0.65 , mientras que la de la lipasa es 0.82 . Los mejores puntos de corte de las curvas fueron $920 \mathrm{U} / \mathrm{l}$ para la amilasa y $1500 \mathrm{U} / \mathrm{l}$ para la lipasa (referencia de laboratorio: lipasa $101 \mathrm{U} / \mathrm{l}$ y amilasa $51 \mathrm{U} / \mathrm{I})$. Únicamente en el caso de la lipasa el resultado es estadísticamente significativo $(p<0.05)$. Los valores diagnósticos de los puntos de corte de la amilasa y de la lipasa se muestran en la tabla 3.

\section{Discusión}

Actualmente, la pancreatitis continúa siendo la complicación más frecuente y temida de la $\mathrm{CPRE}^{14}$. La
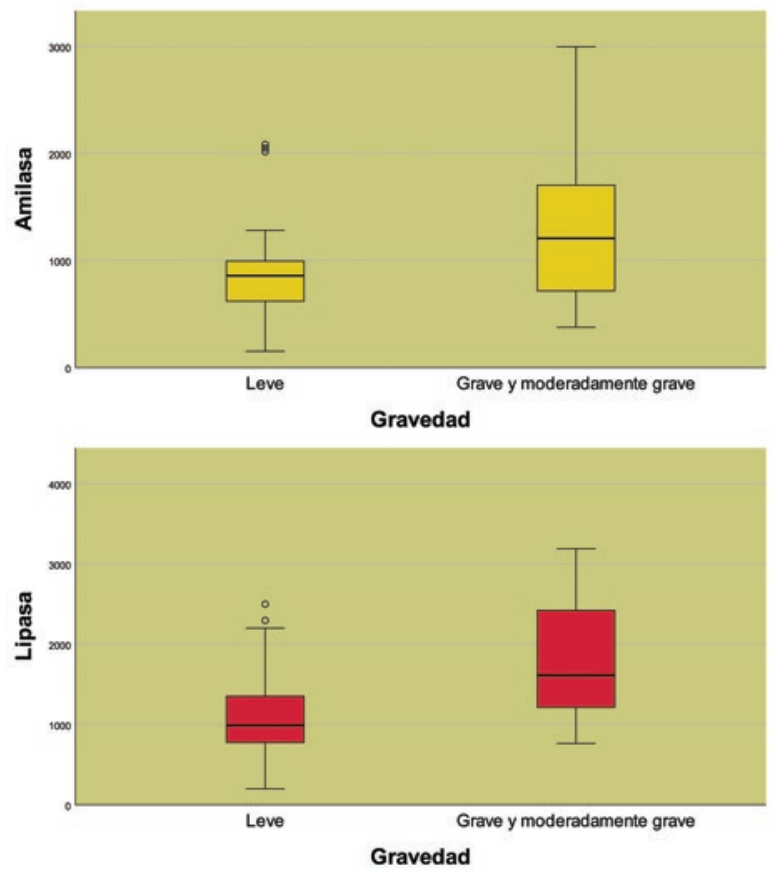

Figura 1. Gráficos que muestran la relación entre las cifras enzimáticas y la gravedad de la pancreatitis poscolangiopancreatografía retrógrada endoscópica. A mayores cifras de amilasa y de lipasa, mayor gravedad de la pancreatitis.

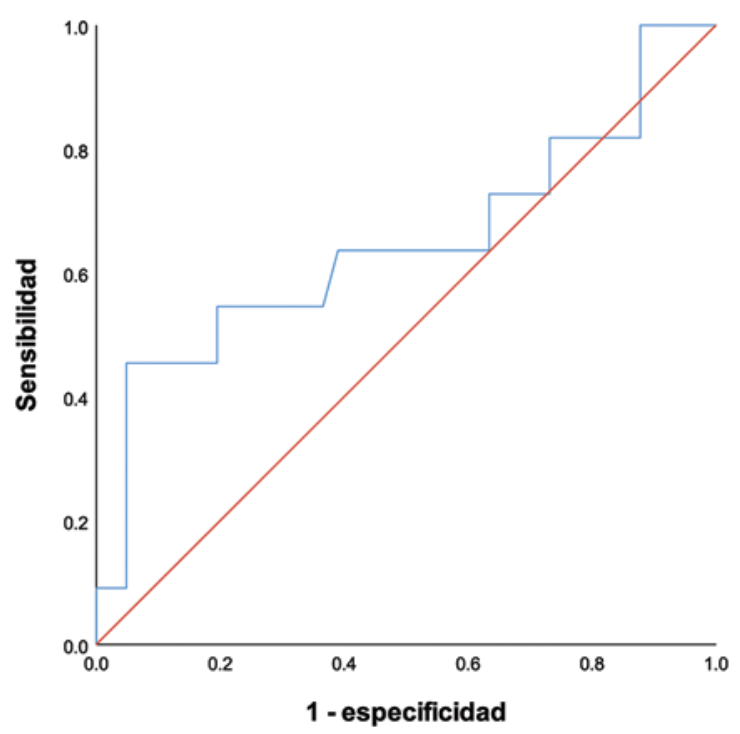

Figura 2. Curva ROC de la amilasa para la predicción de pancreatitis grave y moderadamente grave en pacientes con pancreatitis poscolangiopancreatografía retrógrada endoscópica. Área bajo la curva de 0.65 (intervalo de confianza del 95\%: $0.43-0.86$ ). Mejor valor de corte con sensibilidad del $63 \%$ y especificidad del $67 \%(p=0.13)$.

frecuencia reportada varía ampliamente, desde el $0.5 \%{ }^{15}$ hasta el $40 \%$. En la mayoría de los estudios observacionales y retrospectivos, como este, va del 


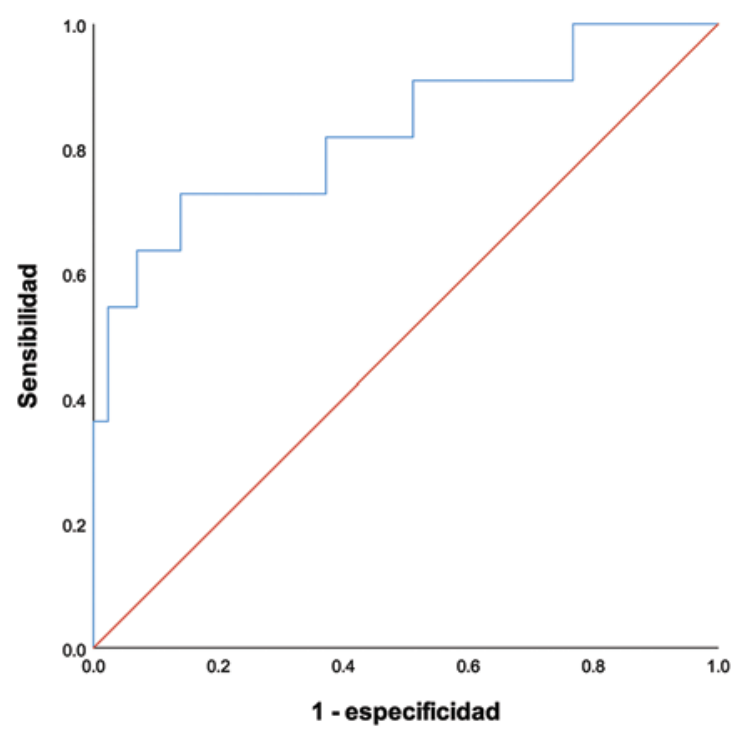

Figura 3. Curva ROC de la lipasa para la predicción de pancreatitis grave y moderadamente grave en pacientes con pancreatitis poscolangiopancreatografía retrógrada endoscópica. Área bajo la curva de 0.827 (intervalo de confianza del 95\%: 0.67-0.98. Mejor valor de corte con sensibilidad del $72.7 \%$, especificidad del $86 \%$ y valor predictivo negativo del $92.5 \%(p<0.05)$.

Tabla 3. Valores diagnósticos de amilasa $920 \mathrm{U} / \mathrm{l}$ y de lipasa $1500 \mathrm{U} / \mathrm{l}$ para la predicción de formas graves de pancreatitis poscolangiopancreatografía retrógrada endoscópica

\begin{tabular}{lccccc}
\hline & \multicolumn{2}{c}{ Amilasa } & & \multicolumn{2}{c}{ Lipasa } \\
\cline { 2 - 3 } \cline { 5 - 6 } & $\%$ & IC $95 \%$ & & $\%$ & IC95\% \\
\hline Sensibilidad & 63.6 & 35.4 a 84.8 & & 72.7 & 43.4 a 90.3 \\
Especificidad & 67.4 & 52.5 a 79.5 & & 86 & 72.7 a 93.4 \\
$\begin{array}{l}\text { Valor predictivo } \\
\text { positivo }\end{array}$ & 33.4 & 17.2 a 54.6 & & 57.1 & 32.6 a 78.6 \\
$\begin{array}{l}\text { Valor predictivo } \\
\text { negativo }\end{array}$ & 87.9 & 72.7 a 95.2 & & 92.5 & 80.1 a 97.4 \\
Exactitud & 66.7 & 53.4 a 77.8 & & 83.3 & 71.3 a 91 \\
Odds ratio & 3.6 & 0.9 a 14 & & 16.4 & 3.4 a 80 \\
\hline IC 95\%: intervalo de confianza del 95\%. & & & & \\
\hline
\end{tabular}

5 al $7 \%{ }^{14}$. En los estudios prospectivos de pacientes no seleccionados se describe del 4 al $8 \%{ }^{3}$. Respecto a lo publicado en México, Montaño, et al. ${ }^{16}$ y Romero, et al. ${ }^{17}$ reportan un $9.4 \%$ y un $6 \%$, respectivamente. En nuestro estudio, la frecuencia fue del $8.7 \%$.

La mayoría de los estudios que investigan la PPCPRE utilizan la definición de consenso y la clasificación de gravedad de Cotton de 1991'; sin embargo, se ha señalado que su uso tiene limitaciones, entre otras porque el grado de gravedad es subjetivo, debido a que en esta clasificación se usa la definición de hospitalización prolongada, que es decidida por el clínico tratante ${ }^{18}$. En nuestro estudio utilizamos la clasificación de pancreatitis de la revisión de Atlanta $^{13}$, que aunque no es específica para PPCPRE $^{1}$ clasifica la gravedad de la pancreatitis de acuerdo con la presencia y la duración de falla orgánica, y las complicaciones locales y sistémicas, en vez de con la duración de la hospitalización ${ }^{19}$. Los estudios recientes sobre pancreatitis aguda utilizan la revisión de Atlanta para clasificar la gravedad, debido a que sus criterios son fácilmente identificables ${ }^{20}$; también en la PPCPRE se ha comenzado a emplear ${ }^{18}$ y es la que mejor refleja su gravedad $^{21}$.

El porcentaje de pacientes con pancreatitis sin dolor abdominal es bajo: el 2.3\% ${ }^{11}$. Encontramos que la presencia de dolor abdominal es altamente sugestiva de diagnóstico de PPCPRE, pero observamos que se llega a presentar en al menos uno de cada cinco pacientes sometidos a CPRE que no desarrollan pancreatitis.

La hiperamilasemia, definida como una elevación de la amilasa de al menos tres veces el límite superior normal sin síntomas de pancreatitis ${ }^{11}$, se presentó en nuestro estudio en el 33.5\%; en la literatura se reporta del 25 al 75\% $\%^{1,11}$. La mayor utilidad de las pruebas de imagen es para la detección de complicaciones, y la mayoría de los pacientes no las requieren para el diagnóstico de pancreatitis aguda ${ }^{22}$; en nuestro estudio solo se ocupó para el diagnóstico en el $0.3 \%$.

Al clasificar a los pacientes por gravedad encontramos porcentajes parecidos a los del estudio de Kim, et al. ${ }^{18}$ sobre factores de riesgo de PPCPRE utilizando la clasificación de Atlanta, en el que se reporta un 83, un 12.8 y un $3.5 \%$ para las pancreatitis leve, moderadamente grave y grave, respectivamente.

La mayoría de los pacientes con pancreatitis presentan episodios leves que se autolimitan ${ }^{1,14}$. Aquellos con episodios moderadamente graves y graves podrían llegar a presentar morbilidad considerable y aumentar la mortalidad ${ }^{4,14}$. Es muy parecido a lo que se reporta para la pancreatitis aguda por otras cau$\operatorname{sas}^{23}$. Sin embargo, el estudio de Ignatavicius, et al. ${ }^{24}$ sugiere que se podría considerar a los pacientes con la forma moderadamente grave dentro del grupo de la PPCPRE leve debido a un curso similar de la enfermedad; sin embargo, en dicho estudio, hasta un $26 \%$ de los pacientes moderadamente graves se comportaron como los graves. 
El uso de las enzimas pancreáticas para predecir la gravedad de la PPCPRE está respaldado por varios estudios. No obstante, los criterios para clasificar la gravedad son diferentes, así como los valores de corte $y$, evidentemente, los grupos de edad 2,6,10-12.

Nuestros resultados confirman que existe correlación entre la elevación enzimática y la presencia y la gravedad de la PPCPRE para el caso de la lipasa sérica, como se reporta en otras publicaciones ${ }^{2,11,25}$ cuyo valor de corte tiene un valor predictivo negativo del $92.5 \%$, por lo que se podrían utilizar las cifras menores de este valor de corte para descartar PPCPRE moderadamente grave o grave. En el caso de la amilasa también existe una correlación menos importante y sin significancia estadística. Nuestros resultados contrastan con lo reportado por varios autores que han publicado valores superiores, tanto de sensibilidad como de especificidad, para un mismo valor de corte y en ambas enzimas ${ }^{6,12,26}$.

Es importante haber llevado a cabo el presente estudio porque nos ofrece información que en nuestro medio hospitalario podría emplearse para la toma de decisiones clínicas en los pacientes sometidos a CPRE, basándose en una prueba disponible. Sin embargo, el estudio presenta algunas limitaciones, como su carácter retrospectivo que impide contar con información más precisa; además, la muestra no es tan grande, lo que podría explicar los amplios intervalos de confianza obtenidos, aunque lo anterior también podría ser causado con menor posibilidad por la baja homogeneidad de la población incluida a pesar de los criterios de inclusión. Se requiere una validación externa en otros hospitales de nuestro país o la realización de estudios prospectivos.

Este estudio es el primero en Latinoamérica que evalúa la utilidad de las enzimas pancreáticas como predictores de gravedad de la PPCPRE.

\section{Conclusiones}

Un valor de la lipasa sérica $>1500 \mathrm{U} / \mathrm{l}$ se correlaciona con la presentación de PPCPRE moderadamente grave y grave, y podría emplearse como herramienta predictiva.

La cuantificación de la lipasa sérica únicamente a los pacientes con presencia de dolor abdominal después de una CPRE parece tener mejor utilidad para predecir formas graves de PPCPRE que la solicitud sistemática de amilasa y lipasa a todos los pacientes sometidos a CPRE. La amilasa sérica muestra una correlación estadísticamente no significativa con las formas graves de PPCPRE.

\section{Agradecimientos}

Al servicio de Endoscopia Gastrointestinal y al Archivo Clínico del Hospital Juárez de México.

\section{Conflicto de intereses}

Los autores declaran no tener conflictos de intereses.

\section{Responsabilidades éticas}

Protección de personas y animales. Los autores declaran que para esta investigación no se han realizado experimentos en seres humanos ni en animales.

Confidencialidad de los datos. Los autores declaran que han seguido los protocolos de su centro de trabajo sobre la publicación de datos de pacientes.

Derecho a la privacidad y consentimiento informado. Los autores han obtenido el consentimiento informado de los pacientes y/o sujetos referidos en el artículo. Este documento obra en poder del autor de correspondencia.

\section{Bibliografía}

1. Chandrasekhar V, Khasha MA, Muthusamy VR, Acosta RD, Agrawal D, Bruining DH, et al. Adverse events associated with ERCP. Gastrointest Endosc. 2017;85:32-47.

2. Nishino T, Toki F, Oyama $H$, Shiratori K. More accurate prediction of post-ERCP pancreatitis by $4-h$ serum lipase levels than amylase levels. Dig Endosc. 2008;20:169-77.

3. Testoni PA, Vailat IC, Giussani A, Notaristefano A, Mariani A. ERCP-induced and non-ERCP-induced acute pancreatitis: two distinct clinical entities with different outcomes in mild and severe form? Dig Liver Dis. 2010;42:567-70.

4. Zitinic I, Plavsic I, Poropat G, Hauser G. ERCP induced and non-ERCP-induced acute pancreatitis: two distinct clinical entities. Medical Hypotheses. 2018;113:42-4.

5. Aziz AMA, Lehman GA. Pancreatitis after endoscopic retrograde cholangiopancreatography. World J Gastroenterol. 2007;13:2655-68.

6. Ito K, Fujita N, Noda Y, Kobayashi G, Horaguchi J, Takasawa O, et al. Relationship between post-ERCP pancreatitis and the change of serum amylase level after the procedure. World J Gastroenterol. 2007;13: 3855-60.

7. Mehta SN, Pavone E, Barkun JS, Bouchard S, Barkun AN. Predictors of post-ERCP complications in patients with suspected choledocholithiasis. Endoscopy. 1998;30:457-63.

8. Friedland S, Soetikno RM, Vandervoot J, Montes H, Tham T. Bedside scoring system to predict the risk of developing pancreatitis following ERCP. Endoscopy. 2002;34:483-8.

9. Sinha A, Cader R, Akshintala VS, Hutfless SM, Zaheer A, Khan VN, et al. Systemic inflammatory response syndrome between 24 and $48 \mathrm{~h}$ after ERCP predicts prolonged length of stay in patients with post-ERCP pancreatitis: a retrospective study. Pancreatology. 2015;15:105-10.

10. Thomas PR, Sengupta S. Prediction of pancreatitis following endoscopic retrograde cholangiopancreatography by the 4-h post procedure amylase level. J Gastroenterol Hepatol. 2001;16:923-6.

11. Concepción-Martín M, Gómez C, Juanes A, Mora J, Vidal S, Diez X, et al. IL-6, IL-10 and TNF do not improve early detection of post-endoscopic retrograde cholangiopancreatography acute pancreatitis: a prospective cohort study. Sci Rep. 2016;6:33492. 
12. Kyung L, Yang M, Kim SS, Noh CK, Cho HJ, Lim SG, et al. Prediction of post-endoscopic retrograde cholangiopancreatography pancreatitis using 4-hour post- endoscopic retrograde cholangiopancreatography serum amylase and lipase levels. J Korean Med Sci. 2017;32:1814-9.

13. Banks PA, Bollen TL, Dervenis C, Gooszen HG, Johnson CD, Sarr MG, et al. Classification of acute pancreatitis - 2012: revision of the Atlanta classification and definitions by international consensus. Gut. 2013;62:102-11.

14. Rustagi T, Jamidar PA. Endoscopic retrograde cholangiopancreatography (ERCP)-related adverse events post-ERCP pancreatitis. Gastrointest Endoscopy Clin N Am. 2015;25:107-21

15. Reyes GA, Suárez LS, Reyes MR, Ríos MA, Rosales JE, Osuna I. Colangiopancreatografía retrógrada endoscópica en un hospital regional del Instituto Mexicano del Seguro Social, 2002-2011: factores de riesgo y complicaciones. Revista de Gastroenterología de México. 2012;77: $125-9$

16. Montaño LA, García CJ, González OA, Fuentes OC, Dávalos CC, Rodríguez LX. Prevención de hiperamilasemia y pancreatitis posterior a la colangiopancreatografía retrógrada endoscópica con la administración rectal de indometacina. Rev Gastroenterol Mex. 2006;71(3):262-8.

17. Romero LA, Torices EE, Domínguez CL, Pantoja C, Rivera NA. Experiencia en la realización de colangiopancreatografía retrógrada endoscópica como procedimiento diagnóstico y terapéutico. Análisis de 1308 casos en 6 años. Rev Gastroenterol Mex. 2006;71(4):149-53.

18. Kim EJ, Cho JH, Yong K, Kim SY, Kim YS. The risk factors for moderately severe and severe post-endoscopic retrograde cholangiopancreatography pancreatitis according to the revised Atlanta Classification. Pancreas. 2017;46:1208-13
19. Kumar A, Griwan MS. A comparison of APACHE II, BISAP, Ranson's score and modified CTSI in predicting the severity of acute pancreatitis based on the 2012 revised Atlanta Classification. Gastroenterol Rep (Oxf). 2018;6:127-31.

20. Arif A, Jaleel F, Rashid K. Accuracy of BISAP score in prediction of severe acute pancreatitis. Pak J Med Sci. 2019;35:1008-12.

21. Smeets X, Bouhouch N, Buxbaum J, Zhang H, Cho J, Verdonk RC, et al. The revised Atlanta criteria more accurately reflect severity of post-ERCP pancreatitis compared to the consensus criteria. United Eur Gastroenterol J. 2019;7:557-64

22. Sahu B, Abbey P, Anand R, Kumar A, Toner S, Malik E. Severity assessment of acute pancreatitis using CT severity Index and modified CT severity Index: correlation with clinical outcomes and severity trading as per the Revised Atlanta Classification. Indiana $\mathrm{J}$ Radiol Imaging 2017;27:152-60.

23. Wang GJ, Gao CF, Wei D, Wang C, Ding SQ. Acute pancreatitis: etiology and common pathogenesis. World J Gastroenterol. 2009;15:1427-30.

24. Ignatavicius P, Gulla A, Cernauskis K, Barauskas G, Dambrauskas Z. How severe is moderately severe acute pancreatitis? Clinical validation of revised 2012 Atlanta Classification. World J Gastroenterol. 2017; 23:7785-90.

25. Papachristos A, Howard T, Thomson BN, Thomas PR. Predicting post-endoscopic retrograde cholangiopancreatography pancreatitis using the 4-h serum lipase level. ANZ J Surg. 2018;88:82-6.

26. Tadehara M, Okuwaki K, Imaizumi H, Kida M, Iwai T, Yamauchi H, et al. Usefulness of serum lipase for early diagnosis of post-endoscopic retrograde cholangiopancreatography pancreatitis. World J Gastrointest Endosc. 2019;11:477-85 